\title{
Extremal throughputs in free-choice nets
}

\author{
Anne Bouillard; Bruno Gaujal
}

August 3, 2005

\begin{abstract}
We give a method to compute the throughput in a timed live and bounded free-choice Petri net under a total allocation (i.e. a 0-1 routing). We also characterize and compute the conflict-solving policies that achieve the smallest throughput in the special case of a 1-bounded net. They do not correspond to total allocations, but still have a small period.
\end{abstract}

\section{Introduction}

Petri nets are logical objects, originally and above all. However, the interest of Petri nets for modelling purposes has induced the need for timed and stochastic extensions of the model. Performance evaluation then becomes a central issue, and the throughput is arguably the main performance indicator.

Consider now a live and bounded free-choice Petri net (LBFC). Such Petri nets realize a good compromise between modelling power and mathematical tractability, see (Desel and Esparza, 1995) for several striking examples of the latter. Assume that the Petri net is timed with a timing specified by a constant real-valued firing time for each transition. To remove the undeterminism of the behavior of the Petri net, a policy for the resolution of all the conflicts needs to be decided. Once it is chosen, all the enabled transitions start to fire as soon as possible, and the time that elapses between the beginning and the completion of the firing of a transition is equal to the firing time. Therefore, the timed evolution of the Petri net is completely determined.

\footnotetext{
*LIP (UMR CNRS, ENS Lyon, INRIA, Université Claude Bernard Lyon 1), École Normale Supérieure de Lyon, 46, allée d'Italie - F-69364 Lyon Cedex 07 - France. Email: anne.bouillard@ens-lyon.fr

${ }^{\dagger}$ Lab. ID-MAG, INRIA-CNRS-UJF-INPG 51, av. J. Kuntzman - F-38330 Montbonnot - France. Email: Bruno.Gaujal@imag.fr

${ }^{\ddagger}$ CNRS-Université Paris 7, LIAFA, Case 7014, 2 place Jussieu - F-75251 Paris cedex 05 - France. Email: Jean.Mairesse@lafa.jussieu.fr
} 
Our goal is to study the global activity or throughput or firing rate of the transitions of the Petri net in a sense to be made precise later on.

This problem has been studied in the context of continuous Petri nets. Computing the throughput given the splitting ratios of "token" at each conflict place has been done in (Recalde and Silva, 2001; Cohen et al., 1998) while the optimization of the splitting ratios for maximizing the throughput is done in (Gaujal and Giua, 2004). These papers use a linear programming method which is not valid in the discrete case, as shown in (Gaujal and Giua, 2004), where the authors also prove that the continuous case provides strict upper bounds on the discrete case. It will become apparent in the following that the discrete case is more intricate.

In the discrete case, the activity depends on the chosen policy for resolving conflicts. In a freechoice Petri net, one may view a conflict-solving policy as a set of local functions associated with conflict places, and assigning tokens to output transitions. The simplest class of policies consists of the so-called 0-1 policies: for a conflict place $p$, allocate all the tokens to a fixed transition. Zero-one policies are called total allocations in (Desel and Esparza, 1995). The next simplest class of policies is, arguably, the periodic ones: for a conflict place $p$, allocate the tokens to the output transitions according to some fixed periodic pattern. Obviously, 0-1 policies are also periodic policies.

In this paper, we address the following natural questions:

- A. Given a periodic policy, is the activity explicitly computable?

- B. Consider the set of all possible, arbitrarily complex, policies for resolving conflicts. Is the infimum, resp. supremum, of the activity over this set attained by a $0-1$ or a periodic policy? Can we explicitly determine the policies realizing the infimum, resp. supremum?

For both questions, we are also concerned by the algorithmic complexity of the computations.

Consider first Question A. It is known that the activity is explicitly computable when the timings are rational-valued (Carlier and Chretienne, 1988). The solution relies on the construction of a very large finite graph $G$ in which a state incorporates three different types of information: the current marking; the remaining time before completion for the currently firing transitions; and the current position of the cursor within the periods for the periodic policy. The timed behavior is ultimately periodic and the period corresponds to an elementary circuit in the graph $G$. The activity is computed along this circuit. 
The method has two major drawbacks. First, it is not efficient from an algorithmic point of view. Indeed, the graph $G$ is in general much larger than the reachability (marking) graph whose size may already be exponential in the one of the Petri net. Second, it does not provide much insight on the structure of the timed behavior.

Here, we show that both restrictions can be overcome in the special case of a 0-1 policy: the live part of the Petri net becomes a disjoint union of event graphs. Consequently, the activity can be computed in polynomial (cubic) time in the size of the Petri net, using classical results on the throughput of timed event graphs (Baccelli et al., 1992; Chretienne, 1983; Cohen et al., 1985). Furthermore, the previous restriction on having rational timings is not necessary anymore for 0-1 policies.

Consider now Question B and assume that the timings are rational-valued for simplicity. Using a simplified version of the above graph $G$, in which the periodic policy is not coded anymore, one can easily prove that the supremum and the infimum of the activity are obtained for periodic policies. The drawbacks are the same as before: the time complexity, and the lack of structural insight. Concerning the latter, the method does not allow to answer the question: is the supremum or infimum attained by $0-1$ policies?

Presumably against the intuition, we exhibit an example of a live and 1-bounded Petri net with firing times all equal to one, and for which the infimum is attained only by non-0-1 periodic policies. More generally, we show that for 1-bounded Petri nets with general $(0, \infty)$-valued timings, the infimum is attained by a periodic policy which may not be $0-1$ but which can be characterized at the net level and which has a very small period (i.e. bounded by the total number of tokens). The same result fails to hold for a $k$-bounded Petri net, $k \geq 2$ where the general structure of infimum policies is not understood. An example is given of a 2-bounded net with timings all equal to one, for which the infimum is attained by periodic policies which are not 0-1 nor have small periods. To be complete, let us mention that the general structure of supremum policies is not well understood, even for 1-bounded nets. It is easy to build examples of 1-bounded LBFC with rational timings for which the supremum is attained only by non-0-1 Sturmian-like periodic policies, as well as examples with irrational timings for which the supremum is attained only by Sturmian-like non-periodic policies, see (Mairesse and Vuillon, 1998) and Example 6.4.

In order to obtain the above results, we use three different types of building blocks:

- The theory of timed event graphs; 
- A structural result stating that the live part of a LBFC with a total allocation is a disjoint union of T-components; and that, given a T-component, there exists a total allocation making this T-component the only live part of the LBFC;

- The notion of Token-Transition invariants. It is a refinement of the classical notion of Tinvariants with a dynamical flavor to it, since it "follows" the evolution of a token.

The first point is very classical (Baccelli et al., 1992; Chretienne, 1983; Cohen et al., 1985), while the other two may be original and of some interest by their own.

The paper is organized as follows. The known results on Question A appear in Section 3. In Section 4 , we study the 0-1 policies in detail. Section 5 introduces the TT-invariants. Section 6 is devoted to Question B. In particular, we characterize the policies which provide the infimum throughput for a 1-bounded net in Subsection 6.3.

A short version, without proofs, of this paper appears in (Bouillard et al., 2005).

\section{Notations and preliminaries}

Denote by $\mathbb{N}$ the nonnegative integers, and by $\mathbb{N}^{*}$ the positive integers. Given a set $T$ and a subset $S$, denote by $\chi_{S}: T \rightarrow\{0,1\}$ the characteristic function of $S$ in $T$ defined by: $\chi_{S}(u)=1$ if $u \in S$ and $\chi_{S}(u)=0$ if $u \in T \backslash S$.

A net is a bipartite directed graph $(\mathcal{P}, \mathcal{T}, \mathcal{F})$ with $\mathcal{P} \cup \mathcal{T}$ as the set of nodes $(\mathcal{P} \cap \mathcal{T}=\emptyset)$ and $\mathcal{F} \subseteq(\mathcal{P} \times \mathcal{T}) \cup(\mathcal{T} \times \mathcal{P})$ as the set of arcs. A Petri net is a quadruple $(\mathcal{P}, \mathcal{T}, \mathcal{F}, M)$, where $(\mathcal{P}, \mathcal{T}, \mathcal{F})$ is a net and $M$ is a map from $\mathcal{P}$ to $\mathbb{N}$. We sometimes write the Petri net $\mathcal{N}$ as $(\mathcal{N}, M)$ to emphasize the special role of $M$. The elements of $\mathcal{P}$ are called places and are represented by circles and those of $\mathcal{T}$ are called transitions and represented by rectangles. The function $M$ is called the (initial) marking of the net and is represented by tokens in places. Let $x \in \mathcal{P} \cup \mathcal{T}$ be a node. We denote by ${ }^{\bullet} x$ the set of its predecessors and by $x^{\bullet}$ the set of its successors. We also set • $X=\cup_{x \in X}{ }^{\bullet} x$ and $X^{\bullet}=\cup_{x \in X} x^{\bullet}$. A transition is conflicting if one of its input place has at least two successors. Otherwise, the transition is non-conflicting.

The marking evolves according to the firing rule. A transition $t$ is enabled if: $\forall p \in \bullet, M(p) \geq 1$. An enabled transition can fire, and then the marking becomes $M^{\prime}$ with $M^{\prime}(p)=M(p)-\chi \cdot{ }_{t}(p)+$ $\chi_{t} \bullet(p)$.

If the marking $M^{\prime}$ is obtained from $M$ by firing the transition $t$, we write $M \stackrel{t}{\rightarrow} M^{\prime}$. If $M^{\prime}$ is obtained by successively firing $\sigma=t_{1} t_{2} \cdots t_{n} \in \mathcal{T}^{*}$, we write $M \stackrel{\sigma}{\rightarrow} M^{\prime}$. The sequence $\sigma$ is called 
ad (admissible) firing sequence. Finally, if $M^{\prime}$ can be reached from $M$ by firing some sequence, we write $M \rightarrow M^{\prime}$. The set of the reachable markings of $M$ is $\mathcal{R}(\mathcal{G}, M)=\mathcal{R}(M)=\left\{M^{\prime} \mid M \rightarrow M^{\prime}\right\}$.

A Petri net is live if for every transition $t$ and every reachable marking $M_{1}$ there exists a marking $M_{2}$, reachable from $M_{1}$, that enables $t$. A Petri net is deadlock-free if there exists no reachable marking in which no transition is enabled. A Petri net is $k$-bounded, $k \in \mathbb{N}$, if for every reachable marking, the number of tokens in a place is less or equal to $k$. A Petri net is bounded if it is $k$-bounded for some $k$. A net $\mathcal{N}$ is structurally live if there exists a marking $M$ such that the Petri net $(\mathcal{N}, M)$ is live. A net $\mathcal{N}$ is well-formed if there is a marking $M$ that makes the Petri net $(\mathcal{N}, M)$ live and bounded

An event graph is a (Petri) net where: $\forall p \in \mathcal{P},|\bullet p|=\left|p^{\bullet}\right|=1$. A state machine is a (Petri) net where: $\forall t \in \mathcal{T},|\bullet t|=\left|t^{\bullet}\right|=1$. A free-choice (Petri) net is a (Petri) net where: $\forall(p, t) \in \mathcal{P} \times \mathcal{T},(p, t) \in \mathcal{F} \Rightarrow\left(p^{\bullet}=\{t\}\right)$ or $(\bullet t=\{p\})$. We use the acronym $L B F C$ for a live and bounded free-choice Petri net. A choice-free (Petri) net is a (Petri) net where: $\forall p \in \mathcal{P},\left|p^{\bullet}\right|=1$.

The incidence matrix of a Petri net is $N \in \mathbb{Z}^{\mathcal{P} \times \mathcal{T}}$ with $N_{p, t}=\chi_{t} \bullet(p)-\chi_{\bullet}(p)$. Let $\sigma \in \mathcal{T}^{*}$ be a firing sequence. The commutative image (or Parikh vector) of $\sigma$ is $\vec{\sigma}=\left(|\sigma|_{t}\right)_{t \in \mathcal{T}}$, the vector of the number of occurrences of each transition $t$ in $\sigma$. If $M \stackrel{\sigma}{\rightarrow} M^{\prime}$, then the equation $M^{\prime}=M+N \vec{\sigma}$ is satisfied.

Invariants of Petri nets. A column vector $J \in \mathbb{N}^{\mathcal{T}} \backslash\left\{(0, \ldots, 0)^{T}\right\}\left(\right.$ resp. $\left.I \in \mathbb{N}^{\mathcal{P}} \backslash\left\{(0, \ldots, 0)^{T}\right\}\right)$ is a $T$-invariant (resp. S-invariant) if $N J=0$ (resp. $\left.I^{T} N=0\right)$. A T-invariant (resp. S-invariant) is minimal if it is minimal for the component-wise ordering among all the T-invariants (resp. S-invariants). A subnet $\mathcal{N}^{\prime}$ of the net $\mathcal{N}$ with the set of nodes $X$ is a $T$-component (resp. $S$ component) if for every transition $t$ of $X,{ }^{\bullet} t \cup t^{\bullet} \subseteq X$ (resp. for every place $p$ of $X,{ }^{\bullet} p \cup p^{\bullet} \subseteq X$ ) and $\mathcal{N}^{\prime}$ is a strongly connected event graph (resp. state machine). If $\left(\mathcal{P}_{1}, \mathcal{T}_{1}, \mathcal{F}_{1}\right)$ is a T-component (resp. S-component) of the net $\mathcal{N}$, then $\chi_{\mathcal{T}_{1}}\left(\right.$ resp. $\left.\chi_{\mathcal{P}_{1}}\right)$ is a minimal T-invariant (resp. S-invariant) of $\mathcal{N}$. For a well-formed free-choice net, the converse is true: if $J$ is a minimal T-invariant (resp. S-invariant), set $\mathcal{T}_{1}=\left\{t \in \mathcal{T} \mid J_{t} \neq 0\right\}$ (resp. $\mathcal{P}_{1}=\left\{p \in \mathcal{P} \mid J_{p} \neq 0\right\}$ ) and $\mathcal{P}_{1}=\bullet \mathcal{T}_{1} \cup \mathcal{T}_{1}^{\bullet}$ (resp. $\left.\mathcal{T}_{1}={ }^{\bullet} \mathcal{P}_{1} \cup \mathcal{P}_{1}^{\bullet}\right)$, then $\left(\mathcal{P}_{1}, \mathcal{T}_{1}, \mathcal{F}_{1}\right)$ is a T-component (resp. S-component). See for instance (Desel and Esparza, 1995, Prop. 5.7, Prop. 5.14, Th. 5.17).

A set of T-components (resp. S-components) forms a T-cover (S-cover) if every node belongs to one of these components. Well-formed free-choice nets are covered by T-components and also by S-components ((Desel and Esparza, 1995, Theorems 6.6 and 5.18)). 
We will also need the following result.

Theorem 2.1. (Desel and Esparza, 1995, Theorem 5.9) Let $p$ be a place of a live and bounded free-choice Petri net $(\mathcal{N}, M)$. The bound of $p$ is $\min \left\{\sum_{s \in \mathcal{P}_{1}} M(s) \mid p \in \mathcal{P}_{1},\left(\mathcal{P}_{1}, \mathcal{T}_{1}, \mathcal{F}_{1}\right)\right.$ is a S-component of $\mathcal{N}\}$.

Clusters. The cluster $[x]$ of $x \in \mathcal{P} \cup \mathcal{T}$ is the smallest subset of $\mathcal{P} \cup \mathcal{T}$ such that: (i) $x \in[x]$; (ii) $p \in \mathcal{P}, p \in[x] \Rightarrow p^{\bullet} \in[x] ;$ (iii) $t \in \mathcal{T}, t \in[x] \Rightarrow \bullet t \in[x]$. The set of all the clusters of a net defines a partition of the nodes of the net. For free-choice nets, each cluster contains only one place or only one transition.

Blocking marking. Let $(\mathcal{N}, M)$ be a Petri net and $t$ a non-conflicting transition of $\mathcal{N}$. A blocking marking of the transition $t$ is a reachable marking such that the only enabled transition is $t$. If $t$ is a conflicting transition, a blocking marking of $t$ is a reachable marking such that the only enabled transitions belong to the cluster $[t]$.

In (Gaujal et al., 2003, Theorem 3.1), it is shown that in a connected LBFC, for any transition $b$, there exists a unique blocking marking $M_{b}$. Moreover, $M_{b}$ is reachable from any other reachable marking without firing $b$.

Timed and routed nets. A timed Petri net is a Petri net in which timings have been added on places and transitions. With no loss of generality, we only consider timings on the transitions, and not on the places. We also consider non-null timings. This is assumed for convenience. The results of the paper could be generalized with null timings, under the assumption that it is not possible to have an infinite number of firings occurring in 0 time. We will come back to this point in Remark 6.3. Set $\mathbb{R}_{+}^{*}=(0,+\infty)$. A timed Petri net is denoted by $(\mathcal{N}, M, \tau)$ with $(\mathcal{N}, M)$ a Petri net and $\tau \in\left(\mathbb{R}_{+}^{*}\right)^{\mathcal{T}}$ the vector of the timings. The timed semantics is the following one. Consider a transition $t$ with timing $\tau_{t}$ which gets enabled at instant $d$. If the transition $t$ is fired, the firing occurs as follows:

- At time $d$, the firing begins. A token is frozen in each input place of $t$ and cannot enable another transition.

- At time $d+\tau_{t}$, the firing ends. The frozen tokens are removed from the input places of $t$ and one token is added in each output place of $t$.

Observe that it is possible for a transition to have several ongoing firings at a given instant. 
The resulting evolution is called as soon as possible (asap), since a firing transition begins to fire as soon as it is enabled.

Any conflict-solving policy may be viewed as a set of local routing functions at each conflicting place. The global routing function is a vector $u=\left(u_{p}\right)_{p \in \mathcal{P}}$ where $u_{p}$ is a function from $\mathbb{N}^{*}$ to $p^{\bullet}$. The $k$-th token arriving in place $p$ (we consider the tokens in place $p$ in the initial marking as the first arriving tokens) can only enable the transition $u_{p}(k)$. So the notion of enabled transition is modified by the routing function. A transition can be fired if all its input places contain a token which is routed to that transition. We denote by $(\mathcal{N}, M, u)$ a routed Petri net with routing $u$ and by $(\mathcal{N}, M, \tau, u)$ a timed and routed Petri net.

A marking is reachable for a routed Petri net $(\mathcal{N}, M, u)$ if it is reachable for $(\mathcal{N}, M)$ via a firing sequence compatible with $u$. The notions of boundedness and liveness of $(\mathcal{N}, M, u)$ are defined accordingly.

If the Petri net $(\mathcal{N}, M)$ is bounded, so is the routed Petri net $(\mathcal{N}, M, u)$. If the Petri net $(\mathcal{N}, M)$ is live, $(\mathcal{N}, M, u)$ is not necessarily live, nor deadlock-free. However, if $(\mathcal{N}, M)$ is a LBFC, the routed net $(\mathcal{N}, M, u)$ cannot have any deadlock, because choices and synchronizations are separated. Hence, if a routed free-choice net is not live, we can always define its non-empty live part.

A routing $u=\left(u_{p}\right)_{p \in \mathcal{P}}$ is periodic if $u_{p}$ is a periodic function for every $p$. A routing $u$ is $0-1$ if: $\forall p \in \mathcal{P}, u_{p}$ is a constant function. A 0-1 routing is called a total allocation in (Desel and Esparza, 1995).

\section{Throughput in routed free-choice Petri nets}

With no loss of generality, all Petri nets considered are assumed to be connected.

Consider a timed Petri net and let $\sigma=\sigma(1) \sigma(2) \cdots \in \mathcal{T}^{\mathbb{N}}$ be an infinite firing sequence. Set $\sigma_{n}=\sigma(1) \cdots \sigma(n)$. Consider the timed evolution starting at instant 0 and associated with $\sigma$. The activity $A(\sigma)$ of $\sigma$ is the asymptotic average number of firings per unit of time:

$$
A(\sigma)=\liminf _{n \rightarrow \infty} \frac{n}{d(n)}
$$

where $d(n)$ is the first instant of completion of all the firings from $\sigma_{n}$.

To make this definition more general and more flexible, it is possible to "weight" the activity of each transition. 
A weight $\alpha_{t} \in \mathbb{R}_{+}$is associated to each transition $t$, we set $\alpha=\left(\alpha_{t}\right)_{t \in \mathcal{T}}$, and we assume that $\alpha \neq(0, \ldots, 0)$. The throughput $D(\sigma)$ of $\sigma$ (for the weight $\alpha$ ) is defined by:

$$
D(\sigma)=\liminf _{n \rightarrow \infty} \frac{\sum_{t \in \mathcal{T}} \alpha_{t}\left(\overrightarrow{\sigma_{n}}\right)_{t}}{d(n)}
$$

If all the weights are equal to one, the throughput is equal to the activity. On the other hand, if $\alpha_{t}=1, \alpha_{t^{\prime}}=0, t^{\prime} \neq t$, then the throughput measures the firing rate of transition $t$.

The above notion of throughput allows one to modify a Petri net without changing its throughput, for example, by replacing a transition of timing $n$ and weight $\alpha$ by $n$ transitions of timing 1 and weight $\alpha / n$.

\subsection{Periodic routings}

Consider a timed and routed $\operatorname{LBFC}(\mathcal{N}, M, \tau, u)$ with a periodic routing and integer firing times (rational firing times can be treated in a similar way).

The state of the Petri net at time $t$ is a triple $\left(M_{t}, R_{t}, U_{t}\right)$ where $M_{t}$ is the marking at time $t, R_{t}$ is the remaining firing time of all the current firings at time $t$ and $U_{t}$ is the current routing decision in all the routing places. Observe that the number of states is finite and bounded by

$(k+1)^{|\mathcal{P}|} \times F^{k|\mathcal{T}|} \times L^{|\mathcal{P}|}$, where $k$ is a bound on the number of tokens per place, $F$ is a bound on the firing times of all the transitions and $L$ is a bound on the period of the routing at each place.

Since the behavior of the net is deterministic, the net jumps from one state to its unique successor at each time-step.

The state space being finite, there exists a state which is visited twice for the first time, and the whole behavior becomes periodic from that point on. This shows that the throughput exists and can be computed along the periodic behavior of the net. However, this computation may have a very high complexity (in time and in space) because the state space is potentially huge.

A construction similar to the above one is proposed in (Carlier and Chretienne, 1988).

\subsection{A particular case: event graphs}

In a live and bounded event graph $(\mathcal{G}, M)$, there is no routing place, hence no routing. In that case, it is useless to sweep the whole state space to compute the throughput. It is well-known that the firing rate is the same for all transitions and the throughput is given by:

$$
D=\frac{\sum_{t \in \mathcal{T}} \alpha_{t}}{\rho(\mathcal{G}, M)}, \quad \text { where } \quad \rho(\mathcal{G}, M)=\max _{c \text { circuit of } \mathcal{G}} \frac{\sum_{t \in c} \tau_{t}}{\sum_{p \in c} M(p)}
$$


The throughput can be computed in cubic time using Karp's algorithm, see for instance (Baccelli et al., 1992). The constant $\rho(\mathcal{G}, M)$ is usually called the cycle time of $(\mathcal{G}, M)$ (see (Baccelli et al., 1992; Chretienne, 1983; Cohen et al., 1985) for details).

\section{Zero-one policies}

In this section, we consider 0-1 routing policies instead of arbitrary periodic routings. We show that all the combinatorial difficulties of periodic routings can be overcome for $0-1$ routings. For modeling purposes, 0-1 routings can also be seen as strict priorities between competing transitions: If one transition has a higher priority than all others transitions in the same cluster, then this corresponds to using a $0-1$ routing sending all tokens to that transition.

\subsection{Total allocations and $0-1$ policies}

An allocation is a function $u$ from a set of clusters $C$ to $\mathcal{T}$ such that: $\forall c \in C, u(c) \in c$. A transition is allocated if it belongs to the image of $u$. An allocation is total if it is defined on all clusters. An allocation points to $C$ if for every place $p$ not belonging to $C$, there exists a path $\pi$ from $p$ to a place of $C$ such that every transition along the path $\pi$ is allocated.

A firing sequence $\sigma$ agrees with an allocation $u: C \rightarrow \mathcal{T}$ if it does not contain any transition $t$ such that $[t] \in C$ and $t \neq u([t])$.

Lemma 4.1. (Desel and Esparza, 1995, Lemma 6.5) Let $C$ be a set of clusters of a strongly connected free-choice Petri net $\mathcal{N}$ and let $\bar{C}$ be the complementary set of $C$ in the clusters of $\mathcal{N}$. Then there exists an allocation $u$ defined on $\bar{C}$ that points to $C$, and if $M$ is a bounded marking and $M \stackrel{\sigma}{\rightarrow}$ is an infinite sequence that agrees with $u$, then some transition of $C$ is fired an infinite number of times in $\sigma$.

In this paper, we see total allocations as $0-1$ routing policies in the places: each place routes all its tokens to its unique allocated output transition.

When $u$ is a $0-1$ routing, $(\mathcal{N}, u)$ is a free-choice Petri net where all the transitions which are not allocated can be removed. Therefore, exactly one output transition remains for each place. We obtain a choice-free Petri net. We first study general choice-free nets before giving a characterization of the choice-free nets obtained as the live part of a free-choice Petri net with a 0-1 routing. 


\subsection{Choice-free nets}

A siphon is a set of places $R$ such that ${ }^{\bullet} R \subseteq R^{\bullet}$. A trap is a set of places $R$ such that $R^{\bullet} \subseteq \bullet$.

Lemma 4.2. A strongly connected choice-free net is structurally live.

Proof. Let $\mathcal{N}$ be a strongly connected choice-free net. Every place has only one output transition. Then, every circuit is a trap. Clearly a siphon of $\mathcal{N}$ contains at least an elementary circuit. If we define the initial marking to be $M: \forall p \in \mathcal{P}, M(p)=1$, then every siphon contains an initially marked trap. By Commoner's Theorem (Desel and Esparza, 1995, Section 4.3), this Petri net is live, so $\mathcal{N}$ is structurally live.

Lemma 4.3. Consider a strongly connected and live choice-free Petri net. It is an event graph if and only if it is bounded.

A connected live and bounded Petri net is strongly connected. So it follows from the above lemma, that a connected and live choice-free Petri net is either unbounded, or bounded in which case it is a strongly connected event graph.

Proof. Let $(\mathcal{N}, M)$ be the choice-free Petri net. Assume that it is bounded. We are in the domain of application of the Blocking Marking Theorem of (Gaujal et al., 2003), recalled in Section 2. We want to show that every place has exactly one input transition. Suppose that there exists a place $p$ with at least two input transitions, $t_{1}$ and $t_{2}$. Let $t$ be the output transition of $p$. Let $M_{t}$ be the blocking marking of $t$. Recall that $M_{t}$ is unique and reachable from $M$, see (Gaujal et al., 2003). From the marking $M_{t}$, let us fire the transition $t$ once and block again the transition $t$ and let the Petri net evolve. The transitions that are fired are well-defined since the net is choice-free. We end up in the marking $M_{t}$ again. Clearly, only one of the transitions $t_{1}$ and $t_{2}$ has been fired. Otherwise there would be one additional token in $p$. Assume it is $t_{1}$ which was not fired. Even if we unblock transition $t$, transition $t_{1}$ will never be fired again. Therefore, the Petri net is not live, which is a contradiction. We conclude that every place has exactly one input transition, meaning that the Petri net is an event graph.

Conversely, assume that $(\mathcal{N}, M)$ is an event graph. Any strongly connected event graph is bounded, which completes the proof.

Figure 1 shows a strongly connected unbounded choice-free net. 


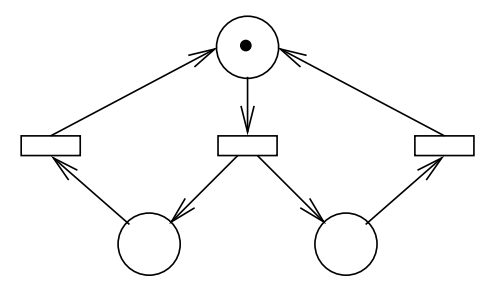

Figure 1: Example of a strongly connected unbounded choice-free net.

\subsection{Live part of a LBFC with a $0-1$ routing}

Consider a $\operatorname{LBFC}(\mathcal{N}, M)$ with a $0-1$ routing. Let $\widetilde{\mathcal{N}}$ be the net obtained by removing all the transitions (together with their input and output arcs) which are not chosen by the $0-1$ routing. This means that the net $\widetilde{\mathcal{N}}$ may not be strongly connected anymore (some places may have no inputs). Figure 4.3 shows the construction of $\widetilde{\mathcal{N}}$ on an example.
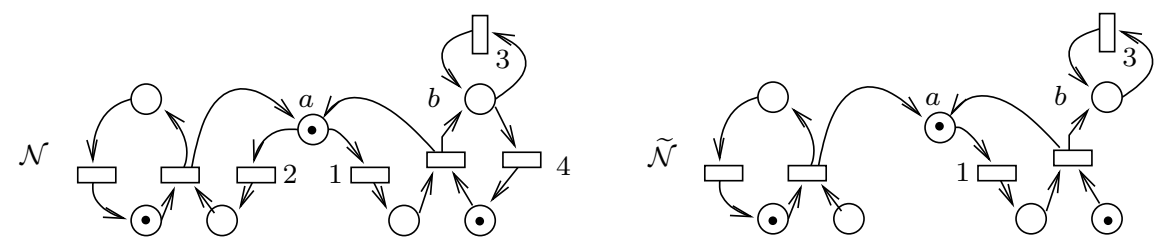

Figure 2: The net $\widetilde{\mathcal{N}}$ is constructed by removing all non-allocated transitions. The $0-1$ routing sends all tokens to transition 1 in routing place $a$ and all tokens to transition 3 in routing place $b$. Transitions 2 and 4 are removed to construct $\tilde{\mathcal{N}}$.

The Petri net $(\widetilde{\mathcal{N}}, M)$ is choice-free and bounded, on the other hand it may not be live. We are interested in characterizing the live part of $(\widetilde{\mathcal{N}}, M)$. This live part may depend on $M$. More precisely, let $M^{\prime}$ be a reachable marking of $(\mathcal{N}, M)$, let $\left(\mathcal{N}_{1}, M_{1}\right)$ be the live part of $(\widetilde{\mathcal{N}}, M)$ and let $\left(\mathcal{N}_{2}, M_{2}\right)$ be the live part of $\left(\widetilde{\mathcal{N}}, M^{\prime}\right)$. We may have $\mathcal{N}_{1} \neq \mathcal{N}_{2}$, as well as $\mathcal{N}_{1}=\mathcal{N}_{2}, \mathcal{R}\left(\mathcal{N}_{1}, M_{1}\right) \neq$ $\mathcal{R}\left(\mathcal{N}_{2}, M_{2}\right)$.

The net $\widetilde{\mathcal{N}}$ can be decomposed into non-trivial maximal strongly connected components (mscc). There are two kinds of such components: the final components and the non-final components.

Lemma 4.4. The final mscc are T-components of $\mathcal{N}$. The non-final mscc are event graphs, but not T-components of $\mathcal{N}$. The final mscc may or may not be live in $\tilde{\mathcal{N}}$, the non-final mscc are not live in $\tilde{\mathcal{N}}$.

Proof. The non-trivial mscc's of $\widetilde{\mathcal{N}}$ are strongly connected choice-free nets. Since the original net is live and bounded, every mscc is bounded. Using both Lemma 4.2 and Lemma 4.3, we deduce 
that all mscc's are event graphs. Let $C$ be a final mscc. We modify the allocation as follows: the allocation in $C$ is not modified; outside of $C$, we choose an allocation that points to $C$. In the resulting choice-free Petri net, there is only one final component which is $C$. From (Desel and Esparza, 1995, Lemma 8.9), for a total allocation, applied from a marking that marks every place, there exists a T-component which is live and where every transition is allocated. This can only be $C$, which is consequently a T-component.

A non-final mscc $C$ is not a T-component. Indeed, since it is non-final and choice-free, there must exist a transition $t \in C$ with an output place $p \notin C$. Moreover, if $C$ was to be live, then the place $p$ could receive an infinite number of tokens, implying that the original Petri net $\mathcal{N}$ is unbounded. So $C$ is not live.

Before proving the main result, Theorem 4.6, we need an additional preliminary lemma.

Lemma 4.5. Let $(\mathcal{G}, M)$ be a live and 1-bounded event graph. Then

- if one token is removed, the event graph is not live anymore,

- if one token is added, the event graph is not 1-bounded anymore.

Proof. Let $p$ be a place such that $M(p)=1$ and $M^{\prime}$ be the marking obtained from $M$ by removing the token of $p$. If $\left(\mathcal{N}, M^{\prime}\right)$ were live, then there would exist a firing sequence $\sigma$ such that $M^{\prime} \stackrel{\sigma}{\longrightarrow} M^{\prime \prime}$ with $M^{\prime \prime}(p)=1$. But this sequence would also be a firing sequence for $(\mathcal{N}, M)$, and there would be 2 tokens in $p$ in the resulting marking. This is a contradiction. Therefore, $\left(\mathcal{N}, M^{\prime}\right)$ is not live.

If a token is added in $(\mathcal{N}, M)$, then the resulting Petri net $\left(\mathcal{N}, M^{\prime}\right)$ is also live. If this token is now removed, then the Petri net is still live (we get the original Petri net $(\mathcal{N}, M)$ ). Therefore, according to the first part of the proof, the Petri net $\left(\mathcal{N}, M^{\prime}\right)$ cannot be 1-bounded.

Theorem 4.6. Let $\left(\mathcal{N}, M_{0}\right)$ be a LBFC with a 0-1 routing. Let $\widetilde{\mathcal{N}}$ be the net obtained from $\mathcal{N}$ by removing the arcs and transitions which are not selected by the routing. The live part of $\left(\widetilde{\mathcal{N}}, M_{0}\right)$ is a non-empty disjoint union of strongly connected event graphs $\left(\mathcal{G}_{1}, M_{1}\right), \ldots,\left(\mathcal{G}_{k}, M_{k}\right)$, where each $\mathcal{G}_{i}$ is a T-component of $\mathcal{N}$. If $\left(\mathcal{N}, M_{0}\right)$ is 1-bounded, or if $k=1$, then the sets of reachable markings $\mathcal{R}\left(\mathcal{G}_{1}, M_{1}\right), \ldots, \mathcal{R}\left(\mathcal{G}_{k}, M_{k}\right)$, do not depend on the 0 -1 routing such that the live part of $\left(\mathcal{N}, M_{0}\right)$ consists of $\mathcal{G}_{1}, \ldots, \mathcal{G}_{k}$.

If $\mathcal{G}$ is a T-component of $\mathcal{N}$, then there exists a 0 -1 routing such that the live part of $\left(\tilde{\mathcal{N}}, M_{0}\right)$ is precisely $(\mathcal{G}, M)$ for some marking $M$.

Proof. It follows from Lemma 4.4 that the live part is $\left(\mathcal{G}_{1}, M_{1}\right), \ldots,\left(\mathcal{G}_{k}, M_{k}\right)$, where each $\mathcal{G}_{i}$ is a T-component. 
Assume that $k=1$ and let us prove that $\mathcal{R}\left(\mathcal{G}_{1}, M_{1}\right)$ does not depend on the 0 -1 routing such that $\mathcal{G}_{1}$ is the live part. In an event graph, the set of reachable markings $\mathcal{R}\left(\mathcal{G}_{1}, M_{1}\right)$ depends only on the number of tokens per circuit. Now, using the uniqueness of the Blocking Marking with respect to $\mathcal{N}$ of a transition of $\mathcal{G}$, we deduce the uniqueness of the total number of tokens on a circuit.

Assume now that $\left(\mathcal{N}, M_{0}\right)$ is 1-bounded. It follows that the event graphs $\left(\mathcal{G}_{i}, M_{i}\right)$ are 1bounded. Now, we show that the reachable markings $\mathcal{R}\left(\mathcal{G}_{i}, M_{i}\right)$ are the same as if $\mathcal{G}_{i}$ was the only live T-component. If there were a circuit of $\mathcal{G}_{i}$ with more tokens than if it were the only live T-component, then $\left(\mathcal{G}_{i}, M_{i}\right)$ would not be 1-bounded, and if there were a circuit with fewer tokens, $\left(\mathcal{G}_{i}, M_{i}\right)$ would not be live, according to Lemma 4.5.

Now fix a T-component $\mathcal{G}$. Let $C$ be the set of clusters of $\mathcal{G}$. Define an allocation that points to $C$, see Lemma 4.1. For the routing places of $\mathcal{N}$ which belong to $\mathcal{G}$, route the tokens within $\mathcal{G}$. Globally this defines a $0-1$ routing such that the Petri net $\widetilde{\mathcal{N}}$ has only one final mscc which is $\mathcal{G}$. Since the non-final mscc are not live, Lemma 4.4, and since the live part of $\widetilde{\mathcal{N}}$ is non-empty (otherwise, it would contradict the liveness of $\mathcal{N}$ ), we conclude that the live part of $\widetilde{\mathcal{N}}$ is precisely $\mathcal{G}$.

Lemma 4.7. In a $L B F C$, every elementary circuit is included in a T-component of the net.

Proof. Let $c$ be an elementary circuit of $\mathcal{N}$, a LBFC, and $u$ a $0-1$ routing such that on $c$, every transition is chosen by the routing, and such that for every other place of choice, the chosen transition is a transition which is on a shortest path to $c$. The choice-free Petri net corresponding to that $0-1$ routing has only one final mscc, so it is the live part of the net. Moreover, this mscc contains $c$. The live part of the net is a T-component, so $c$ is included in that T-component.

\subsection{Throughput of 1 -bounded free-choice nets with 0 -1 routings}

We now have all the ingredients to prove the main result.

Theorem 4.8. Consider a $\operatorname{LBFC}\left(\mathcal{N}, M_{0}\right)$ with a 0 -1 routing. Assume that the live part is $(\mathcal{G}, M)$, where $\mathcal{G}$ is a single $T$-component corresponding to the T-invariant $J_{\mathcal{G}}$. Then the throughput does not depend on the 0-1 routing such that $\mathcal{G}$ is the live part, and it is equal to

$$
D_{\mathcal{G}}=\frac{\alpha^{T} J_{\mathcal{G}}}{\rho(\mathcal{G}, M)}
$$


Assume that the live part is $\left(\mathcal{G}_{1}, M_{1}\right), \cdots,\left(\mathcal{G}_{k}, M_{k}\right)$, where $\mathcal{G}_{i}$ is a T-component. Then the throughput is

$$
D=\sum_{i=1}^{k} D_{\mathcal{G}_{i}}=\sum_{i=1}^{k} \frac{\alpha^{T} J_{\mathcal{G}_{i}}}{\rho\left(\mathcal{G}_{i}, M_{i}\right)} .
$$

If the free-choice Petri net is 1-bounded, then this throughput does not depend on the 0-1 routing such that the live part consists of $\mathcal{G}_{1}, \ldots, \mathcal{G}_{k}$.

Proof. The proof easily follows from (1) and Theorem 4.6. Observe that the cycle time $\rho(\mathcal{G}, M)$ of an event graph depends on $M$ only through the token count of circuits, see (1).

\section{Token-Transition-Invariants}

Let $(\mathcal{N}, M)$ be a Petri net. Let $\sigma=\sigma_{1} \cdots \sigma_{k}$ be a firing sequence. We say that $\sigma^{\prime}=\sigma_{1}^{\prime} \cdots \sigma_{h}^{\prime}$, $h \leq k$ is a subsequence of $\sigma$ if there exists an increasing function $f:\{1, \ldots, h\} \rightarrow\{1, \ldots, k\}$ such that $\sigma_{i}^{\prime}=\sigma_{f(i)}$ for all $i \in\{1, \ldots, h\}$.

Definition 5.1 (compatible firing sequence). Let $\pi=p_{1} t_{1} p_{2} t_{2} \cdots p_{\ell} t_{\ell}, p_{i} \in \mathcal{P}, t_{i} \in \mathcal{T}$ be a path of $\mathcal{N}$ and $M$ be a marking that marks $p_{1}$. Let $\sigma$ be an admissible firing sequence of $(\mathcal{N}, M)$. The sequence $\sigma$ is compatible with $\pi$ if the first subsequence of $\sigma$ in $\left[t_{1}\right]\left[t_{2}\right] \cdots\left[t_{\ell}\right]$ is $t_{1} t_{2} \cdots t_{\ell}$.

In the above definition, first has the following meaning. Order all subsequences of $\sigma$ according to the point-wise ordering of the increasing functions used to defined them $(f \leq g$ is $f(i) \leq g(i)$ for all $i$ in the domain of $f$ ). First means smallest according to this ordering.

In other words, an admissible firing sequence $\sigma$ is compatible with a path in the Petri net if all the transitions along that path are fired and in that order when $\sigma$ is fired. This means that the token which was initially in place $p_{1}$ successively enters places $p_{2}, \ldots, p_{\ell}$ when $\sigma$ is fired.

Definition 5.2 (Token-Transition-Invariant). Let $c=p_{1} t_{1} p_{2} t_{2} \cdots p_{\ell} t_{\ell}$ be a circuit of $\mathcal{N}$ and $M$ be a marking that marks $p_{1}$. A vector $J \in \mathbb{N}^{\mathcal{T}}$ is a Token-Transition-invariant (or TTinvariant) generated by $c$ and the marking $M$ if it is a T-invariant and if it is the commutative image of an admissible firing sequence compatible with $c$.

A TT-invariant $J$ generated by $c$ and the marking $M$ is minimal if for every other TT-invariant $J^{\prime}$ generated by $c$ and the marking $M, J^{\prime}$ is not smaller than $J$.

A TT-invariant generated by $c$ is minimal if for every other TT-invariant $J^{\prime}$ generated by $c$ and some marking, $J^{\prime}$ is not smaller than $J$. 
In words, a TT-invariant is a T-invariant such that one token has moved along a circuit and is back to its original place when the corresponding sequence is fired (hence the name).

In spite of what the definition suggests, TT-invariants generated by $c$ do not depend on the initial marking: if the commutative image of $\sigma_{1} \cdot \sigma_{2}$ is a TT-invariant for $c$, so is the commutative image of $\sigma_{2} \cdot \sigma_{1}$, since it is a firing sequence from the marking $M^{\prime}$ such that $M \stackrel{\sigma_{1}}{\rightarrow} M^{\prime}$.

However, unlike general T-invariants, TT-invariants depend on the set of reachable markings. We will see in the following that they actually mainly depend on the maximal possible number of tokens in circuit $c$.

The following Lemma characterizes minimal TT-invariants in event graphs, where things are easy.

Lemma 5.3. Let $c$ be an elementary circuit of a live and 1-bounded event graph containing $n$ tokens. The minimal TT-invariant generated by $c$ is $(n, \ldots, n) \in \mathbb{N}^{\mathcal{T}}$.

Proof. Recall that the T-invariants of an event graph are of the form $(x, \ldots, x), x \in \mathbb{N} \backslash\{0\}$, see (Desel and Esparza, 1995, Prop. 3.16). Now, let $J$ be a minimal TT-invariant associated with $c=p_{1} t_{1} \cdots$, and let $\sigma$ be a corresponding compatible firing sequence. Since the Petri net is 1-bounded, tokens along the circuit $c$ cannot overtake each other. Hence, when the token initially in $p_{1}$ is back to $p_{1}$, after the firing of $\sigma$, we know that transition $t_{1}$ must have fired $n$ times.

Example 5.4. Figure 3 shows the evolution of an event graph containing 2 tokens in circuit c. We look at the minimal TT-invariant generated by c. Using Lemma 5.3, the minimal TT-invariant is $(2,2,2,2,2,2,2,2,2)$. The white token (as well as the black one) is back to its original place (Figure 3(c)).

The minimal T-invariant is $(1,1,1,1,1,1,1,1,1)$. Note that, after a single firing of every transition (Figure 3(b)), the marking is unchanged, but the white token has switched its position with the black one. After firing every transition again (Figure 3(c)), the white token is back in the right place.

We now characterize the minimal TT-invariants generated by a circuit of a live and 1-bounded free-choice Petri net. This is a more difficult case.

Let us first give an algorithm to build every minimal TT-invariant (Lemma 5.5 and Proposition 5.6). The following results (Lemmas 5.7, 5.8 and Proposition 5.9) show that a minimal TTinvariant generated by a circuit $c$ is the sum of at most $n$ minimal T-invariants, where $n$ is the maximal number of tokens that $c$ may contain. 

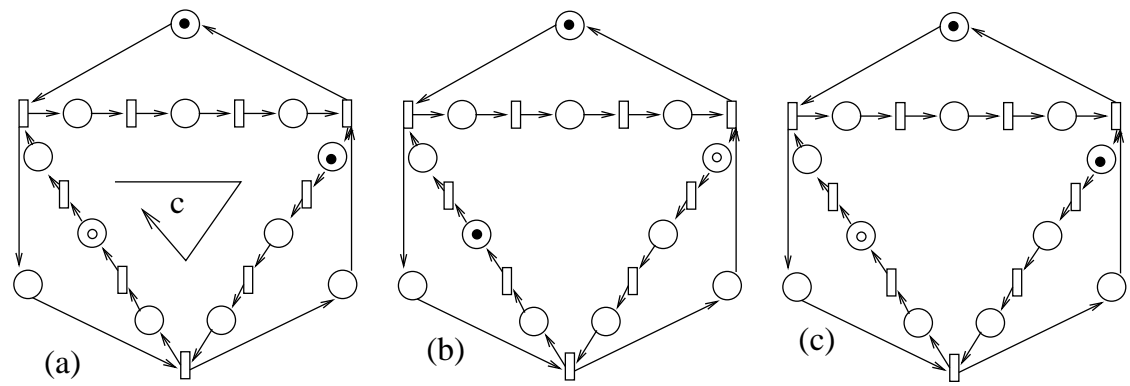

Figure 3: TT-invariant in an event graph

The proofs of the following results use the following notions. Let $\sigma$ be an admissible firing sequence containing transition $b$. We consider $\left.\sigma\right|_{b}$ the longest subsequence of $\sigma$ which is an admissible firing sequence and which does not contain $b$. This uniquely defines $\left.\sigma\right|_{b}$. Intuitively, $\left.\sigma\right|_{b}$ is the subsequence of $\sigma$ to be actually fired in a timed version of the Petri net where the firing time of transition $b$ is set to $+\infty$. Let $\left.\sigma \backslash \sigma\right|_{b}$ be the complementary sequence of $\left.\sigma\right|_{b}$ in $\sigma$. Let $M^{\prime}$ be the marking obtained after firing $\left.\sigma\right|_{b}$. In $M^{\prime}$, among all the transitions to appear in $\left.\sigma \backslash \sigma\right|_{b}$, transition $b$ is the only one to be enabled.

Lemma 5.5. Let $c$ be a circuit of $\mathcal{N}$ a live and 1-bounded free-choice net, and $b$ be a transition of c. Recall that $M_{b}$ is the unique blocking marking associated with b. For every minimal TTinvariant $J$, there exists a firing sequence $\sigma$ compatible with $c$ such that $J=\vec{\sigma}$ that can be fired from $M_{b}$.

Proof. Let $J$ be a minimal TT-invariant generated by $c$ and $\sigma$ a firing sequence such that $\vec{\sigma}=J$, and enabled from a reachable marking $M$. From $M$, the firing sequence $\sigma$ is admissible. Let $b$ be a transition of $c$. Let $M^{\prime}$ be such that $M \stackrel{\left.\sigma\right|_{b}}{\rightarrow} M^{\prime}$. Let Cluster $\left(\left.\sigma \backslash \sigma\right|_{b}\right)$ be the set of the clusters of all the transitions appearing in $\left.\sigma \backslash \sigma\right|_{b}$. Now, let us show that there exists a firing sequence leading from $M^{\prime}$ to $M_{b}$ and which does not use the transitions of Cluster $\left(\left.\sigma \backslash \sigma\right|_{b}\right)$. Let $\sigma_{1}$ be a firing sequence from $M^{\prime}$ to $M_{b}$ and let $t$ be the first transition (if it exists) of $\sigma_{1}$ that belongs to Cluster $\left(\left.\sigma \backslash \sigma\right|_{b}\right)$. Then, there exists an input place $p$ of $t$, that is marked during the firing of $\sigma_{1}$. But that place can also be marked independently by firing $\left.\sigma \backslash \sigma\right|_{b}$. But then, $p$ is not 1-bounded. Consequently, the marking $M_{b}$ can be reached without firing the transitions of $\operatorname{Cluster}\left(\left.\sigma \backslash \sigma\right|_{b}\right)$, which ends the proof of the lemma.

Proposition 5.6. Let $c$ be a circuit of $\mathcal{N}$, a live and 1-bounded free-choice net. Every minimal TT-invariant generated by c can be found by applying Algorithm 1. 


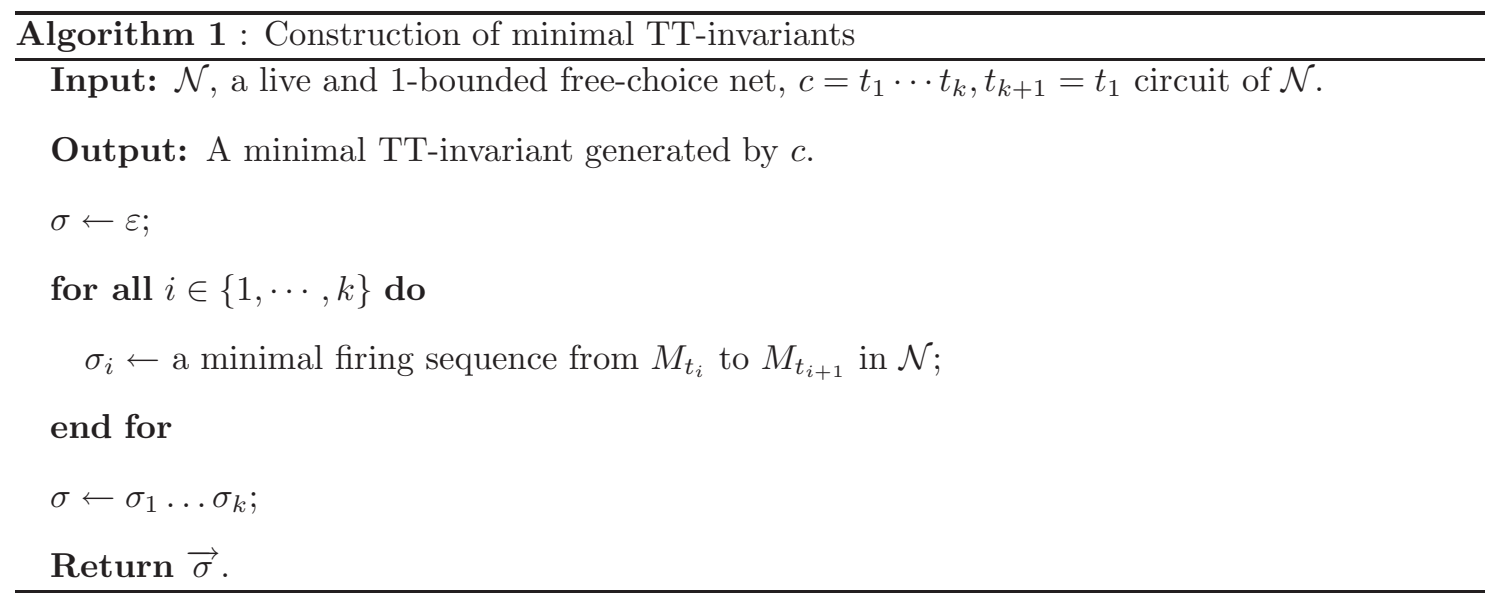

In the algorithm, a firing sequence $\sigma: M \stackrel{\sigma}{\rightarrow} M^{\prime}$ is a minimal firing sequence if it does not contain any subsequence $\sigma^{\prime}: M \stackrel{\sigma^{\prime}}{\rightarrow} M^{\prime}$. Such a minimal firing sequence has no reason to be unique. Hence the algorithm may yield several different outputs for a given input.

Proof. By construction, the output of Algorithm 1 is a TT-invariant generated by $c$. Let $J$ be a minimal TT-invariant generated by $c$. From Lemma 5.5, there exists $\sigma \in \mathcal{T}^{*}$ such that $\vec{\sigma}=J$ and $M_{t_{1}} \stackrel{\sigma}{\rightarrow} M_{t_{1}}$. Now, consider the maximal admissible subsequence $\left.\sigma\right|_{t_{2}}$. Firing $\left.\sigma\right|_{t_{2}}$ leads from $M_{t_{1}}$ to a marking $M_{2}$ in which no transition from $\left.\sigma \backslash \sigma\right|_{t_{2}}$ can fire except $t_{2}$.

If $M_{2}$ is not $M_{t_{2}}$, this means that there exists a transition $r \neq t_{2}$ which is enabled under $M_{2}$. By definition of $\left.\sigma\right|_{t_{2}}$, this transition will always remain enabled through the firing of $\left.\sigma \backslash \sigma\right|_{t_{2}}$. The final marking is $M_{t_{1}}$ in which $t_{1}$ is the only enabled transition. Therefore $r=t_{1}$.

Now, from $M_{2}$, let us fire the longest admissible subsequence of $\left.\sigma \backslash \sigma\right|_{t_{2}}$ not containing $t_{3}$. We reach $M_{3}$ where no transition can fire except $t_{3}$ and $t_{1}$, using the same reasoning as before. By repeating the argument, we reach $M_{k}$ in which only $t_{k}$ and $t_{1}$ may be fired. Finally, firing $t_{k}$ brings one more token in the place between $t_{k}$ and $t_{1}$ contradicting one-boundedness.

This means that all the way through, $t_{1}$ has not been enabled. The successive markings $M_{2}, \cdots, M_{k}$ are indeed the blocking markings $M_{t_{2}}, \cdots, M_{t_{k}}$.

We now show that a minimal TT-invariant generated by an elementary circuit is the sum of $n$ minimal T-invariants, where $n$ is the maximal number of tokens in $c$ (it is given by the number of tokens in $c$ under the blocking marking of any transition of $c$ ). Lemma 5.7 and Lemma 5.8 are technical lemmas used to prove Proposition 5.9.

Lemma 5.7. Let $c=t_{1} \cdots t_{k} t_{k+1}=t_{1}$ be an elementary circuit of a live and 1-bounded free-choice net $\mathcal{N}$. For every $i \in\{1, \cdots, k\}$ and for every minimal firing sequence $\sigma$ from $M_{t_{i}}$ to $M_{t_{i+1}}$, there 
exists a minimal T-invariant $J$ such that $\chi_{\left\{t_{i}, t_{i+1}\right\}} \leq J$ and $\vec{\sigma} \leq J$.

Proof. In this proof, we set $t=t_{i}$ and $t^{\prime}=t_{i+1}$. There exists a T-component $C$ containing $t$ and $t^{\prime}$ by Lemma 4.7 .

Case (a): Let $\sigma$ (resp. $\sigma^{\prime}$ ) be an admissible minimal firing sequence from $M_{t}$ to $M_{t^{\prime}}$ (resp. from $M_{t^{\prime}}$ to $M_{t}$ ) using only transitions in $C$. Then $\overrightarrow{\sigma \sigma^{\prime}}$ is a minimal T-invariant: it is a T-invariant and $t$ and $t^{\prime}$ are fired only once, hence all the other transitions of $C$ also, using elementary properties of T-invariants in event graphs. Note that this implies that $\sigma^{\prime}$ contains any transition at most once.

Case (b): Let $\sigma$ be a minimal firing sequence from $M_{t}$ to $M_{t^{\prime}}$ (with transitions not necessarily in $C$ ). Let $\sigma^{\prime}$ be a minimal firing sequence from $M_{t^{\prime}}$ to $M_{t}$ containing only transitions of $C$. Using case (a), $\sigma^{\prime}$ contains any transition at most once: $\overrightarrow{\sigma^{\prime}} \leq \chi_{C}$. The commutative image $J$ of $\sigma \cdot \sigma^{\prime}$ is also a T-invariant. Let $p$ be the place of the circuit $c$ between $t$ and $t^{\prime}$. We have $\sum_{a \in p} \cdot J_{a}=1$ : indeed, transitions of $p^{\bullet}$ do not appear in $\sigma$ by minimality of $\sigma$, and only $t^{\prime}$ appears, once, in $\sigma^{\prime}$. Therefore, $J$ is a T-invariant containing $t$ and $t^{\prime}$ only once.

Now consider $J_{1}$ a minimal T-invariant included in $J$ and containing $t$ and $t^{\prime}$. Using case (a), $J_{1}=\overrightarrow{\sigma_{1} \sigma_{1}^{\prime}}$ with $M_{t} \stackrel{\sigma_{1}}{\rightarrow} M_{t^{\prime}} \stackrel{\sigma_{1}^{\prime}}{\rightarrow} M_{t}$. Since $\sigma^{\prime}$ and $\sigma_{1}^{\prime}$ are both minimal sequences, none is strictly included in the other. If they are not equal, there exists a transition $a_{1} \in \sigma_{1}^{\prime} \backslash \sigma^{\prime}$. Therefore, the T-invariant $\overrightarrow{\sigma_{1} \sigma^{\prime}}$ does not contain $a_{1}$ but contains $t$ and $t^{\prime}$ once. Let us now consider the minimal T-invariant $J_{2}$ included in $\overrightarrow{\sigma_{1} \sigma^{\prime}}$ and containing $t$ and $t^{\prime}$. Using case (a) again, $J_{2}=\overrightarrow{\sigma_{2} \sigma_{2}^{\prime}}$ with $M_{t} \stackrel{\sigma_{2}}{\rightarrow} M_{t^{\prime}} \stackrel{\sigma_{2}^{\prime}}{\rightarrow} M_{t}$. Again, $\sigma^{\prime}$ and $\sigma_{2}^{\prime}$ are both minimal sequences, none is strictly included in the other. If they are not equal, there exists a transition $a_{2} \in \sigma_{2}^{\prime} \backslash \sigma^{\prime}$. This means that the T-invariant $\overrightarrow{\sigma_{2} \sigma^{\prime}}$ does not contain $\left\{a_{1}, a_{2}\right\}$. The construction goes on as long as $\sigma_{i}^{\prime}$ does not coincide with $\sigma^{\prime}$ but must stop after a finite number of iterations. We finally get $\sigma_{i}^{\prime}=\sigma^{\prime}$. Now, $\sigma_{i} \sigma^{\prime}=\sigma_{i} \sigma_{i}^{\prime} \subseteq \sigma \sigma^{\prime}$. Therefore, $J-J_{i}=\overrightarrow{\sigma \sigma^{\prime}}-\overrightarrow{\sigma_{i} \sigma^{\prime}}$ is a T-invariant included in $\vec{\sigma}$. Since $\sigma$ is a minimal sequence, this has to be empty. Therefore, $J=J_{i}$ is a minimal T-invariant.

Lemma 5.8. Let $c$ be an elementary circuit of $\mathcal{N}$, a live and 1-bounded free-choice net and $n$ be the maximal number of tokens in $c$. There exists a minimal TT-invariant generated by $c$ that is $n$ times the same minimal T-invariant.

Proof. By Lemma 4.7, there exists a T-component $C$ containing the circuit $c$. By Theorem 4.6, there exists a 0-1 routing under which the live part of the Petri net is precisely $C$. In the resulting marking $M_{C}, c$ contains $n$ tokens, according to Theorem 4.6. Let $\mathcal{T}_{C}$ be the set of transitions of $C$. 
Applying Lemma 5.3 to the event graph $\left(C, M_{C}\right)$, we obtain that $n \chi_{\mathcal{T}_{C}}$ is a minimal TT-invariant generated by $c$.

Proposition 5.9. Let $c$ be an elementary circuit of $\mathcal{N}$, a live and 1-bounded free-choice net and $n$ be the maximal number of tokens in c. Every minimal TT-invariant generated by $c$ is the sum of $n$ minimal T-invariants.

Proof. From Lemma 5.8, there exists a minimal TT-invariant generated by $c$ which is the sum of $n$ minimal T-invariants. Let $J$ be such a TT-invariant which is equal to $n K, K$ being a minimal T-invariant corresponding to a T-component containing the circuit $c$. Let $\sigma$ be the firing sequence such that $\vec{\sigma}=J$ corresponding to the concatenation of firing sequences $\sigma_{t_{i} t_{i+1}}$ from the blocking marking of $t_{i}$ to the blocking marking of $t_{i+1}$ found in Algorithm 1.

Using Proposition 5.6, we know that all minimal TT-invariants can be obtained by replacing each $\sigma_{t_{i} t_{i+1}}$ by another minimal firing sequence. The proof holds by induction on the number of replacements used to get any given TT-invariant. We prove by induction that all TT-invariants obtained are sums of $n$ T-invariants and that for all $i, \sigma_{t_{i} t_{i+1}}$ belongs to one T-invariant in the sum.

This is true at the beginning, since $J=n K$ and $\vec{\sigma}_{t_{i} t_{i+1}} \leq K$ for all $i$.

Let $t$ and $t^{\prime}$ be two consecutive transitions and replace the original sequence $\sigma_{t t^{\prime}}$ by another minimal firing sequence from $M_{t}$ to $M_{t^{\prime}}, \sigma_{t t^{\prime}}^{\prime}$. A new minimal TT-invariant $J^{\prime}$ generated by $c$ is then obtained. By induction, the original sequence $\sigma_{t t^{\prime}}$ belongs to one minimal T-invariant, say $H$, involved in the sum forming $J$. One has $H=\overrightarrow{\sigma_{t t^{\prime}} \cdot w}$, where $M_{t^{\prime}} \stackrel{w}{\rightarrow} M_{t}$. Using the proof of Lemma 5.7, $L=\overrightarrow{\sigma_{t t^{\prime}}^{\prime} w}$ is still a minimal T-invariant. The new TT-invariant is $J^{\prime}=J-H+L$. It is still a sum of $n$ T-invariants. Furthermore, all the other sequences $\sigma_{v v^{\prime}}$, with $\left(v, v^{\prime}\right) \neq\left(t, t^{\prime}\right)$, still belong to a single T-invariant (those in $H$ are now in $L$, no change for all the others). This ends the proof.

Example 5.10. Figure 4 illustrates Proposition 5.9: consider the circuit c $=1,2,3,5,6,7,9,10,11$. The blocking marking of transition 1 is $\{d, g, k\}$. To reach the blocking marking of transition 2, the two possible firing sequences are 1,9,10,11 or 1,9,12. To reach the blocking marking of transition 3, 2 is fired, and to reach the blocking marking of transition 5, 3 is fired. By symmetry of the net, there are also two possible minimal firing sequences from $M_{5}$ to $M_{6}$, that are $5,1,2,3$ or $5,1,4$ and from $M_{9}$ to $M_{10}, 9,5,6,7$ or 9,5,8. Every other minimal firing sequence from a blocking marking to the next on circuit $c$ is made of only one transition. Then, there 
are 8 minimal TT-invariants (for three subsequences, there are two possibilities). For example, $1,9,10,11,2,3,5,1,4,6,7,9,5,8,10,11$ is a firing sequence compatible with c whose commutative image is a minimal TT-invariant, which can be written as $I+J$, where $I$ and $J$ are minimal T-invariants, of respective support 1,2,3,5,6,7,9,10,11 and 1,4,5,8,9,10,11.

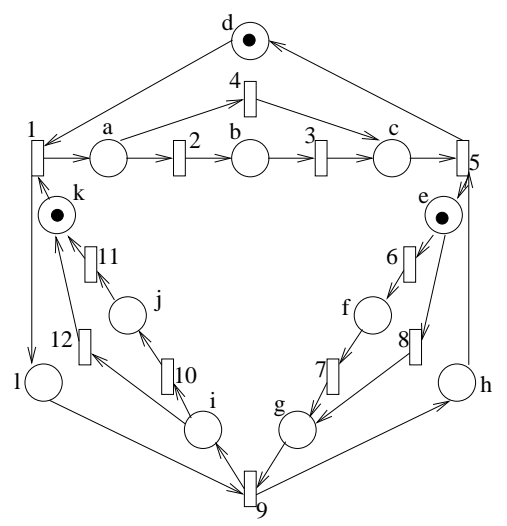

Figure 4: Example of a free-choice net.

Lemma 5.11. Let $\mathcal{C}$ be a circuit in a live and 1-bounded free-choice net and $J$ be a minimal TT-invariant of $\mathcal{C}$. The circuit is composed of the elementary circuits $c_{1}, \ldots, c_{k}$. Then there exists $k$ minimal TT-invariants, $J_{1}, \ldots, J_{k}$ respectively generated by $c_{1}, \ldots, c_{k}$ such that $J=\sum_{i=1}^{k} J_{i}$.

Proof. This is an immediate consequence of Proposition 5.6.

\section{$6 \quad$ Extremal Throughputs}

We now consider all possible, arbitrarily complex, routing policies and try to address the following questions: Can one compute the routing policy which yields the best or worst throughput? Are the best and worst policies periodic? Are the best and worst policies 0-1?

\subsection{Dominance of periodic policies}

In this section we show that the best and worst throughputs in LBFC with rational firing times are achieved by periodic policies, and we provide an algorithm to construct them. The construction is very close to the one given in Section 3.1 and is basically the one in (Carlier and Chretienne, 1988).

Consider a timed LBFC with non-null rational timings $\left(\tau_{t}\right)_{t \in \mathcal{T}}$ and with a vector of weights $\alpha=\left(\alpha_{t}\right)_{t \in \mathcal{T}}$, see Section 3. Let $x \in \mathbb{Q}$ be such that $\tau_{t}=k_{t} x, k_{t} \in \mathbb{N} \backslash\{0\}$, for all $t \in \mathcal{T}$. Build a 
new LBFC by replacing each transition by a path: a transition with timing $\tau_{t}$ and weight $\alpha_{t}$ is replaced by $k_{t}$ transitions of timing 1 and weight $\alpha_{t} /\left(k_{t} x\right)$. Consider an infinite firing sequence $\sigma$ for the original Petri net and the corresponding firing sequence $\sigma^{\prime}$ in the new Petri net. Then the throughputs, defined as in Section 3, associated with $\sigma$ and $\sigma^{\prime}$ coincide. Therefore we can, without loss of generality, consider LBFC with timings all equal to 1.

\subsubsection{Asap marking graph of a free-choice net.}

Consider a timed $\operatorname{LBFC}(\mathcal{N}, M, \tau)$ and assume that all the timings are equal to $1: \forall t \in \mathcal{T}, \tau_{t}=1$. Denote by $N$ the incidence matrix. In a given marking, a transition may be enabled several times. So the transitions that can be fired simultaneously form a multi-set.

The asap marking graph is defined as follows:

$Q \leftarrow\{M\} ; \operatorname{Arc} \leftarrow \emptyset ; \tilde{Q} \leftarrow\{M\} ;$

while $\tilde{Q} \neq \emptyset$ do

Pick $M^{\prime} \in \tilde{Q}$;

for all maximal multi-set $U$ of transitions that can be fired simultaneously from $M^{\prime}$ do

$$
\begin{aligned}
& M^{\prime \prime} \leftarrow M^{\prime}+N . U ; \\
& \text { if } M^{\prime \prime} \notin Q \text { then } \\
& \quad Q \leftarrow Q \cup\left\{M^{\prime \prime}\right\} ; \tilde{Q} \leftarrow \tilde{Q} \cup\left\{M^{\prime \prime}\right\} ;
\end{aligned}
$$

end if

$\operatorname{Arc} \leftarrow \operatorname{Arc} \cup\left\{M^{\prime} \rightarrow M^{\prime \prime}\right.$, with label and weight $\left.\left[U \mid \sum_{t \in U} \alpha_{t}\right]\right\} ;$

end for

$\tilde{Q} \leftarrow \tilde{Q} \backslash\left\{M^{\prime}\right\}$

end while

The above construction stops because the Petri net is bounded. So the asap marking graph is finite.

All the as soon as possible (asap) evolutions of the Petri net can be read on this graph, hence its name. In this graph, the weight of a path is the sum of the weights of the arcs. The average weight of a path is its weight divided by its length (number of arcs).

Theorem 6.1. Let $(\mathcal{N}, M, \tau)$ be a timed LBFC with non-null rational timings. The minimal and maximal throughputs are obtained for periodic routings.

Proof. Let $(\tilde{\mathcal{N}}, \widetilde{M})$ be the LBFC obtained from $(\mathcal{N}, M)$ after duplicating the transitions such that 
every transition in the new Petri net has timing 1. Consider the asap marking graph of $(\widetilde{\mathcal{N}}, \widetilde{M})$. Let $c$ be a circuit of the asap graph of maximum average weight. The maximal throughput can be reached by following this circuit.

Also, the minimal throughput can be reached by following the circuit of minimal average weight.

This shows that the corresponding routings are periodic. Indeed the routing can be deduced from the labels along the circuit of the asap marking graph. In particular, the period is smaller than the length of the circuit.

Example 6.2. Consider the Petri net of Figure 4. Every transition has timing 1 and weight 1. The asap marking graph is represented in Figure 5.

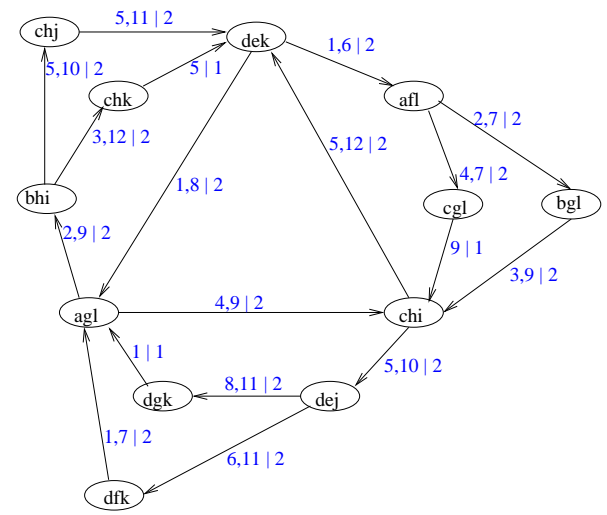

Figure 5: Asap marking graph corresponding to the Petri net of Figure 4.

The minimal average weight of a circuit is $15 / 9$, given by the circuit $\{(d e k),(a f l),(c g l),($ chi $)$, $(d e j),(d g k),(a g l),(b h i),(c h k)\}$. This gives the minimal throughput and the routing to reach it. The maximal average weight is attained for the circuit $\{($ dek $),($ agl $),($ chi $)\}$. And the maximal throughput is 2 .

When the graph is built, computing the throughput can be made in cubic time in the number of nodes of the asap marking graph. But this graph can have an exponential size in the size of the original Petri net. One reason is that the number of markings of the net can be exponential in the size of the net. The other reason is that transitions are duplicated to build the Petri net with timings 1 starting from a Petri net with rational timings. Moreover, this method gives no information about the structure of the corresponding extremal policies.

Remark 6.3. If we allow timings that are rational but possibly null (on the condition that there exists no infinite firing sequence of timing zero), the asap marking graph is constructed as follows. 
We first construct a new LBFC with firing times 0 or 1 exactly as above. Now, let $M$ and $M^{\prime}$ be two reachable markings. There is an arc from $M$ to $M^{\prime}$ if there exists a maximal set of transitions $S$ enabled under $M$ such that

1. all of them have timing one and

2. $M^{\prime}$ can be reached from $M$ by firing all the transitions in $S$ followed by an arbitrary number of transitions of timing 0 .

The arcs are labeled accordingly.

\subsection{Non-rational timings}

For general non-rational timings, the maximal throughput is not always periodic, even for onebounded nets, as shown in the following example.

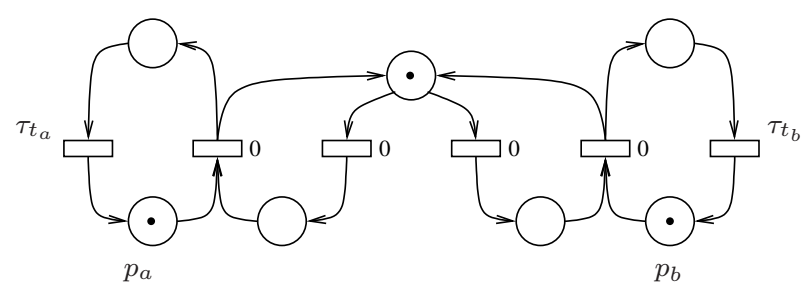

Figure 6: One-bounded Petri net for which the optimal routing is not periodic.

Example 6.4. Look at Figure 6. The value of the firing times are given next to the transitions on the Figure. We have $\tau_{t_{a}} / \tau_{t_{b}} \notin \mathbb{Q}$. This model has been studied in (Mairesse and Vuillon, 1998). The optimal routing is Sturmian aperiodic. The best routing consists in choosing the left $\left(t_{a}\right)$ or right transition $\left(t_{b}\right)$ depending on whether one token appears first in $p_{a}$ or in $p_{b}$. If $t_{a}$ has fired $n_{a}$ times and $t_{b} n_{b}$ times, it suffices to compare $n_{a} \tau_{a}$ and $n_{b} \tau_{b}$. The non-periodicity comes from the irrationality of the ratio of the timings.

The same Petri net can be considered with non-null rational timings approximating the ones in Figure 6.4. In this case, the maximal throughput is achieved for a Sturmian-like periodic routing policy. In particular, it is not possible to give an absolute bound for the period.

The above behaviors contrast sharply with the results to be proved in Section 6.3 on the minimal throughput for 1-bounded nets.

For general non-rational timings, we do not know if the minimal throughput is always attained by periodic policies. In the next section, we show however that it is the case for the subclass of 1-bounded Petri nets. 


\subsection{Minimal throughput in 1-bounded free-choice nets}

Consider a timed live and 1-bounded free-choice net $(\mathcal{N}, M, \tau)$. In this part, we show that the minimal throughput is obtained for a periodic routing even for general non-rational timings. Furthermore, we give a precise insight on the structure of the periodic routing reaching the minimal throughput. Roughly it corresponds to a critical TT-invariant.

From Theorem 4.8, we can easily deduce the following lemma:

Lemma 6.5. The worst 0-1 routing can be chosen among those that make only one T-component live.

Critical circuit. Suppose again that the timings are 1. The worst routing can be read on the asap marking graph by considering an elementary circuit, $c=\left(M_{0}, \cdots, M_{T-1}\right)$, of minimal average weight. The length of $c$ is $T$, the period of the evolution.

Let $t_{0}$ be a live transition of the net appearing in the label between states $M_{0}$ and $M_{1}$ of the circuit $c$. We build a path in the Petri net with final extremity $t_{0}$ in the following recursive way.

If the path $t_{i}, \cdots, t_{1}, t_{0}$ is built, we choose the transition $t_{i+1}$ in the label $U_{i}$ of the arc between $M_{T-i-1[T]}$ and $M_{T-i[T]}$ and such that $t_{i+1} \in{ }^{\bullet} t_{i}$.

We stop the construction when we find $m \in \mathbb{N}^{*}$ and a transition $t_{j}$ such that $t_{j}=t_{j-m T}$. Consider the circuit of the Petri net corresponding to the sequence of transitions $t_{j}, t_{j-1}, \ldots, t_{j-m T+1}$, and denote it by $\mathcal{C}_{c}$. The length of this circuit is $m T$ by construction.

Let $U_{i}$ be the set of transitions whose firing leads from $M_{T-i-1[T]}$ to $M_{T-i[T]}$. (Here $U_{i}$ is a set and not a multi-set, because the Petri net is 1-bounded.)

Let $K$ be the commutative image of $\sigma=U_{j-1} \cdots U_{j-m T}$. By construction, $K$ is a T-invariant. Also by construction, $\left(t_{j-1} \cdots t_{j-m T}\right)$ is the first sub-word of $\sigma$ belonging to $\left[t_{j-1}\right], \ldots,\left[t_{j-m T}\right]$. So, $K$ is a TT-invariant generated by $\mathcal{C}_{c}$.

Since $K$ is a T-invariant associated with a firing sequence following a minimal weight circuit in the asap marking graph, we deduce that the worst throughput $\underline{D}$ of $(\mathcal{N}, M, \tau)$ satisfies:

$$
\underline{D}=\frac{\alpha^{T} K}{m T} .
$$

Since $K$ is a TT-invariant for $\mathcal{C}_{c}$, there exists $J_{c}$ a minimal TT-invariant for $\mathcal{C}_{c}$ such that $K \geq J_{c}$. The circuit $\mathcal{C}_{c}$ may not be elementary. In full generality, it is composed of, say, $k$ elementary circuits $c_{1}, \cdots, c_{k}$ of length $\ell_{1}, \cdots, \ell_{k}$, with $\sum_{i=1}^{k} \ell_{i}=m T$. By Lemma 5.11, we have $J_{c}=\sum_{i=1}^{k} J_{i}$ where $J_{i}$ is a minimal TT-invariant of $c_{i}$. Then, 


$$
\underline{D}=\frac{\alpha^{T} K}{m T} \geq \frac{\alpha^{T} J_{c}}{m T}=\frac{\alpha^{T}\left(\sum_{i=1}^{k} J_{i}\right)}{\sum_{i=1}^{k} \ell_{i}}=\frac{\sum_{i=1}^{k} \alpha^{T} J_{i}}{\sum_{i=1}^{k} \ell_{i}} \geq \min _{i=1}^{k} \frac{\alpha^{T} J_{i}}{\ell_{i}} .
$$

Now, by definition, $\underline{D} \leq \min _{i=1}^{k}\left(\alpha^{T} J_{i}\right) / \ell_{i}$. Therefore, we can find an elementary circuit $c$ of the Petri net, of length $\ell$ and of associated minimal TT-invariant $J$, such that $\underline{D}=\alpha^{T} J / \ell$. Such a circuit is called a critical circuit of the Petri net.

We are now ready to prove the main result of this section. Set $\mathbb{R}_{+}^{*}=(0,+\infty)$.

Theorem 6.6. Consider a timed live and 1-bounded free-choice net with general timings in $\mathbb{R}_{+}^{*}$. Let $\alpha$ be a weight on the transitions. The minimal throughput is obtained for a periodic routing. For each place, the period of the routing function is bounded by the maximal number of tokens in the net.

Proof. Assume first that the timings are rational and non-null. Then, we can consider without loss of generality (as seen before), that all the timings are equal to 1 . Let us denote by $\tau(c)$ the sum of the timings along the circuit $c$ (here, it is also the number of transitions of $c$ ).

Let $c$ be a critical circuit of the Petri net. In particular, $c$ is an elementary circuit. By Proposition 5.9, a minimal TT-invariant generated by $c$ is always of the form

$$
J=\sum_{i=1}^{n} J_{i}, \quad J_{i} \text { minimal T-invariant }
$$

where $n$ is the maximal number of tokens in $c$.

Then, each place is marked at most $n$ times when a sequence of commutative image $J$ is fired. But $n$ is less than the total maximal number of tokens in $\mathcal{N}$. This completes the proof in the rational case.

Now let us prove the result for timings in $\mathbb{R}_{+}^{*}$. We first prove the following lemma.

Lemma 6.7. Let $(\mathcal{N}, u, \tau)$ be a routed and timed live and 1-bounded free-choice net. Let $\alpha$ be a weight on the transitions and consider the throughput with respect to this weight. The throughput is a continuous and non-increasing (for the coordinate-wise ordering) function of the timings $\left(\sigma_{t}\right)_{t \in \mathcal{T}}$ in the region $\left(\mathbb{R}_{+}^{*}\right)^{\mathcal{T}}$.

Proof. We use here the representation of 1-bounded free-choice nets by heaps of pieces, see (Gaubert and Mairesse, 1999). Since the net is routed, the resulting infinite firing sequence is defined up to commutation between concurrent transitions, and it is independent of the timings. Consider one of the possible infinite firing sequences and pile up the pieces one by one in the 
order given by the firing sequence. We denote by $H_{n}$ the height of the heap after the piling of the first $n$ pieces, and by $A_{n}$ the corresponding total weight. The throughput $D$ is defined by: $D=\lim _{n \rightarrow \infty} A_{n} / H_{n}$.

We change the timing of $t_{0}$ from $\sigma_{t_{0}}$ to $\sigma_{t_{0}}+\varepsilon, \varepsilon>0$. Let $D(\varepsilon)$ be the new throughput. After the piling of $n$ pieces, the weight is still $A_{n}$, and the height of the new heap is $H_{n}^{\prime} \leq H_{n}+K_{n} \varepsilon$ where $K_{n}$ is the number of occurrences of $t_{0}$ in the first $n$ pieces. We have $D(\varepsilon)=\lim _{n \rightarrow \infty} A_{n} / H_{n}^{\prime}$. Since $A_{n} / H_{n} \geq A_{n} / H_{n}^{\prime}$, we have $D \geq D(\varepsilon)$. Moreover, we have,

$$
0 \leq \frac{A_{n}}{H_{n}}-\frac{A_{n}}{H_{n}^{\prime}} \leq \frac{A_{n}}{H_{n}}-\frac{A_{n}}{H_{n}+K_{n} \varepsilon} \leq \frac{A_{n} K_{n} \varepsilon}{H_{n}\left(H_{n}+K_{n} \varepsilon\right)} \leq \frac{A_{n} K_{n} \varepsilon}{H_{n}^{2}} .
$$

Let $\underline{\sigma}$ be the minimal firing time of a transition. Let $C$ be the number of columns in the heap model (which is equal to the number of places in the Petri net, see (Gaubert and Mairesse, 1999)). We have $H_{n} \geq n \underline{\sigma} / C$. Observe also that $K_{n} \leq n$. We deduce that:

$$
\frac{A_{n}}{H_{n}}-\frac{A_{n}}{H_{n}^{\prime}} \leq \frac{A_{n} C \varepsilon}{H_{n} \underline{\sigma}} .
$$

By going to the limit in $n$, we get $D-D(\varepsilon) \leq \operatorname{Cst} D \varepsilon$. In particular, $\lim _{\varepsilon \rightarrow 0} D(\varepsilon)=D$. The same type of argument applies if we change the timing of $t_{0}$ from $\sigma_{t_{0}}$ to $\sigma_{t_{0}}-\varepsilon>0, \varepsilon>0$. This completes the proof.

Continuation of the proof of Theorem 6.6.

Denote by $\underline{D}:\left(\mathbb{R}_{+}^{*}\right)^{\mathcal{T}} \rightarrow \mathbb{R}_{+}^{*}$, the infimum over all possible routings of the throughput, viewed as a function of the timings. Clearly, $\underline{D}$ is non-increasing. Consider $x \in\left(\mathbb{R}_{+}^{*}\right)^{\mathcal{T}}$. We approach $x$ with a sequence of rational tuples $\left(x^{(k)}\right)_{k \in \mathbb{N}}$ satisfying $\forall k \in \mathbb{N}, x^{(k)} \geq x$. Hence we have: $\forall k, \underline{D}\left(x^{(k)}\right) \leq \underline{D}(x)$.

Let $m$ be the maximal number of tokens in a circuit of the free-choice net $\mathcal{N}$. When changing the timings, $m$ remains the same. For rational timings, we know that the minimal throughput is reached for a periodic routing whose period is at most $m$ (first part of the proof). There are only a finite number of these routings. So there exists a subsequence $\left(x^{(\phi(k))}\right)$ of timings for which the corresponding minimal throughput is reached for the same periodic routing $r$. Now denote by $D(x, r)$ the throughput corresponding to the timings $x$ and the periodic routing $r$. Using Lemma 6.7, we have $D(x, r)=\lim _{k \rightarrow \infty} \underline{D}\left(x^{(\phi(k))}\right)$. It follows that $D(x, r) \leq \underline{D}(x)$ and we conclude that $D(x, r)=\underline{D}(x)$. This completes the proof.

Example 6.8. Consider again the example of Figure 4. Form the corresponding asap marking graph shown on Figure $5,(1,2,3,5,6,7,9,10,11)$ is the critical circuit. Since every transition has 
weight 1, the firing sequences of minimal weight from the blocking marking of a transition of $c$ to the blocking marking of the next transition of c are (starting from the blocking marking of transition 1 which is $\{d, k, g\}):(1,9,12)$; $(2) ;(3) ;(5,1,4) ;(6) ;(7) ;(9,5,8) ;(10) ;(11)$. The minimal TTinvariant achieving the minimal throughput is composed of two minimal T-invariant of support $1,2,3,5,6,7,9,10,11$ and $1,4,5,8,9,12$. The minimal throughput is then $15 / 9$ as anounced before. As for the worst routing policy, it can be obtained directly from the minimal TT-invariant: $u_{a}=$ $(2,4)^{\infty}, u_{e}=(8,6)^{\infty}$ and $u_{i}=(12,10)^{\infty}$.

\subsection{Algorithm to compute a routing that minimizes the throughput}

Consider a timed live and 1-bounded free-choice net $(\mathcal{N}, M, \tau)$. Let $N$ be the incidence matrix. Let Clusters be the set of clusters. Define the matrix $K \in\{0,1\}^{\text {Clusters } \times \mathcal{T}}$ such that $K_{a, t}=1$ if $t$ belongs to the cluster $a$ and $K_{a, t}=0$ otherwise.

Let $S$ be a subset of $\mathcal{T}$. Let Lightest-T-invariant $(S)$ be the algorithm that computes a minimal T-invariant of minimal weight that contains the transitions of $S$. It is the solution of the following linear programming problem:

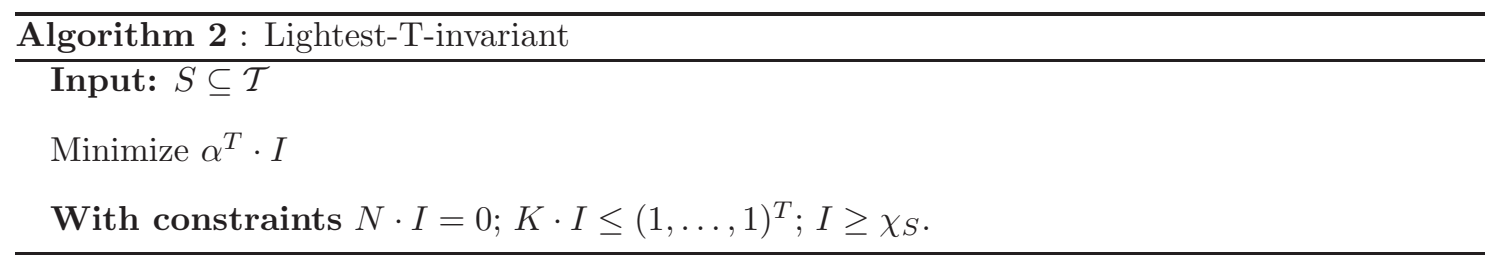

When the algorithm is called when $S$ is a set of transitions belonging to the same T-component, the condition $K \cdot I \leq(1, \ldots, 1)^{T}$ ensures that the output is a set of disjoint minimal T-invariants, with only one containing $S$.

This algorithm runs in polynomial time since it is a linear program over $\mathbb{Q}$. Due to the form of the constraints, the solution will always be $\{0,1\}$-valued.

Let us define the functions Blocking-Marking $(t)$, Cycle-time $(A)$, and Timing $(c)$.

- Blocking-Marking $(t)$ computes the blocking marking of transition $t$. This marking can be computed in time $O\left(|\mathcal{T}|^{2}\right)$ when the Petri net is an event graph, and in time $O\left(|\mathcal{T}|^{3}\right)$ for the general free-choice case, see (Gaujal et al., 2003).

- Cycle-time $(A)$ computes the cycle time of a $(\max ,+)$ matrix $A$. Here, it is used for matrices of dimension at most $|\mathcal{P}|$. Then, the time complexity is $O\left(|\mathcal{P}|^{3}\right)$ using Karp's algorithm, see for instance (Baccelli et al., 1992). 
- Timing $(c)$ computes the sum of the firing times of the transitions along the circuit. The time complexity is linear.

In the algorithm below, the $(\max ,+)$ representation of the behavior of live and 1-bounded free-choice nets is used. For every transition $b, A_{b}$ is the $(\max ,+)$ matrix representing the time behavior of the firing of $b$ (see (Gaubert and Mairesse, 1999) for more details). The symbol $\otimes$ denotes the multiplication of matrices in the $(\max ,+)$ algebra. This operation can be done in cubic time in the dimension of the matrices. Here, it is used for matrices of dimension at most $|\mathcal{P}|$.

Using the previous results, we get the following theorem.

Theorem 6.9. Algorithm 3 finds the minimal throughput of a timed live and 1-bounded free-choice Petri net.

At each iteration in the first loop, the time complexity is $O\left(|\mathcal{T}|^{3}\right)$, and there are $|\mathcal{T}|$ iterations. Consider now the second loop. There are at most $|\mathcal{T}|^{2}$ iterations in the loop. At each iteration, we need to find a minimal sequence from $M_{b}$ to $M_{b^{\prime}}$ in a T-component. This can be done in time $O\left(|\mathcal{T}|^{2}\right)$. The length of a minimal sequence is of order $O\left(|\mathcal{T}|^{2}\right)$, see (Gaujal et al., 2003). Hence the matrix $A_{b b^{\prime}}$ can be computed in time $O\left(|\mathcal{P}|^{3}|\mathcal{T}|^{2}\right)$. At each iteration of the last loop, the time complexity is $\left.O\left(|\mathcal{P}|^{3}|\mathcal{T}|\right)\right)$ and there are as many iterations as elementary circuits in the net. Therefore, the total time complexity is $O\left(\mathcal{C}|\mathcal{P}|^{3}|\mathcal{T}|\right)$, where $\mathcal{C}$ is the number of elementary circuits. Since the number of elementary circuits can be exponential in the number of places $\left(O\left(2^{|\mathcal{T}|}\right)\right)$, the time complexity is exponential in the worst case. As for the space complexity, it remains polynomial in the size of the Petri net.

For comparison, consider the method of computation given (for rational timings) in Section 6.1.1 and which uses the asap marking graph. The size of the asap marking graph is exponential in the size of the original Petri net, more precisely its size is $O\left(2^{|\mathcal{P}|}\right)$. So the complexity in time is $O\left(\left(2^{|\mathcal{P}|}\right)^{3}\right)=O\left(8^{|\mathcal{P}|}\right)$ and the space complexity is at least $O\left(2^{|\mathcal{P}|}\right)$. Observe that these complexities are evaluated without taking into account the necessity of transforming the Petri net with rational timings into an equivalent one with timings equal to 1, see Section 6.1.1. This transformation makes both time and space complexity of the classical method even worse.

Finally, remark that our construction also gives some insight on the period of the worse policy. Since the critical TT-invariant associated with the critical circuit (with $n$ tokens) is the sum of $n$ T-invariants, this means that the period of the worse routing policy in each routing place is 


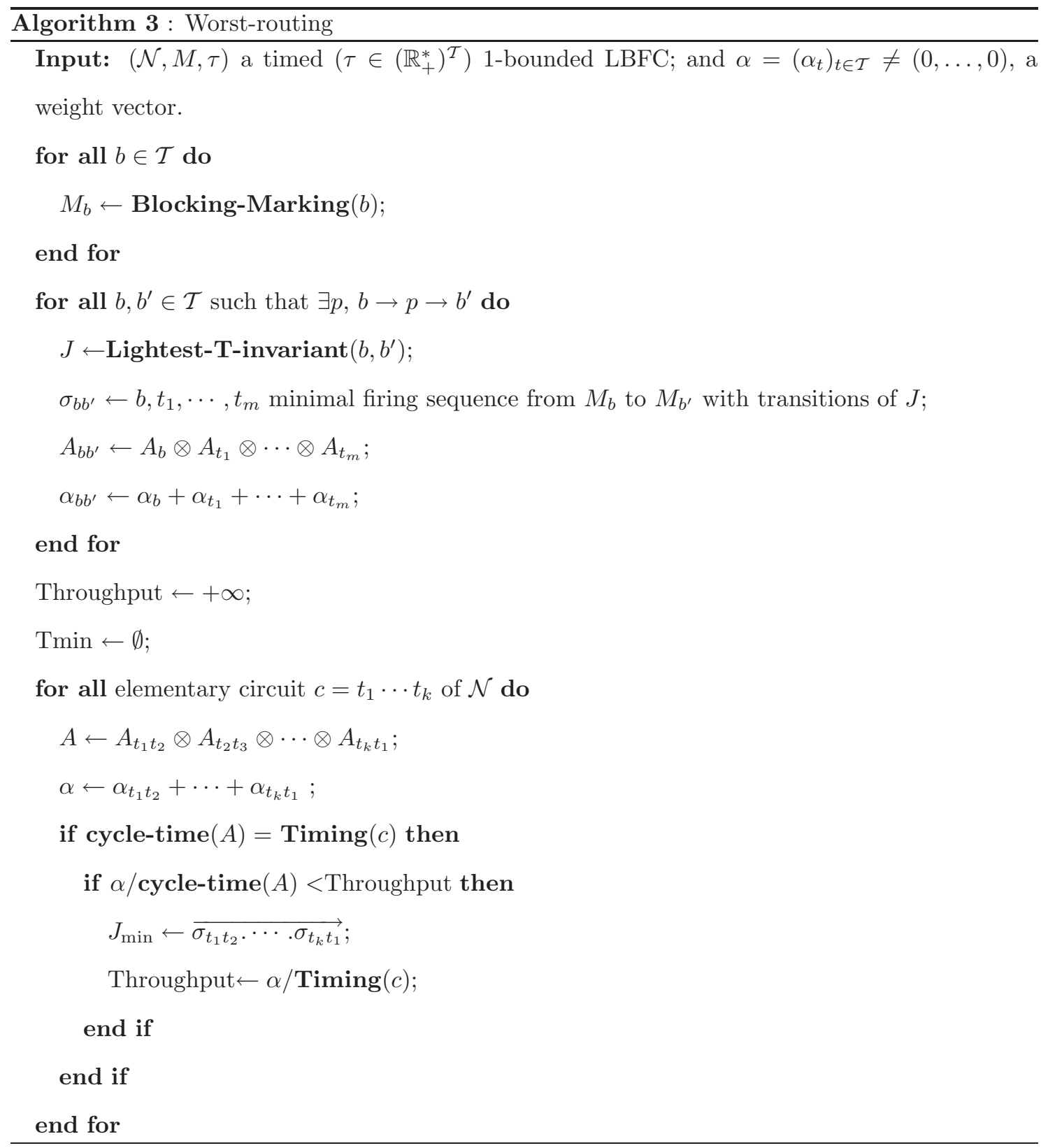


periodic with a period $n \leq|\mathcal{P}|$. This is several order of magnitude smaller than the period that can be deduced from the classical algorithm which is exponential $O\left(2^{|\mathcal{P}|}\right)$.

\subsection{Bounded nets}

If one considers a live and $k$-bounded free-choice net with $k \geq 2$, then the previous constructions for 1-bounded nets do not work anymore. If the timings are rational, the worst throughput is reached for periodic routings (Theorem 6.1), but the period is not bounded by the number of tokens in a circuit, as shown by the following example.

Example 6.10. Figure 7(a) represents a 2-bounded free-choice net where the period of the worst routing is greater that the number of tokens in any circuit. Furthermore the critical circuit for this routing is not elementary.

(a)

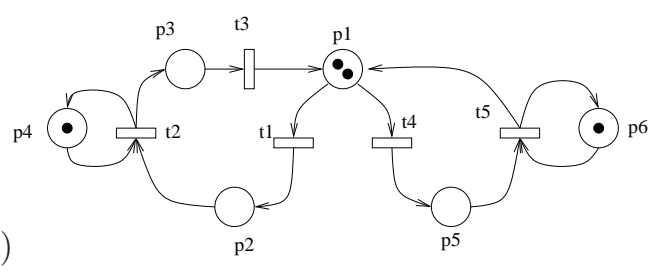

(b)

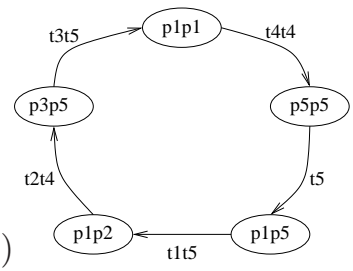

Figure 7: Free-choice net and its worst evolution.

All the timings are equal to 1 , as well as the weights. The routing in $p_{1}$ which gives the minimal throughput is, $(t 1 t 4 t 4 t 4)^{\infty}$, and the periodic evolution is given in Figure $7(b)$.

The throughput of this evolution is 9/5, whereas if only the left (or right) event graph is live the throughput is 2. The period of the routing function of place $p_{1}$ is greater than the number of tokens in the circuits containing this place (2 tokens), as opposed to what happens in the case of 1-bounded nets. In this example, by changing the timings and/or increasing the number of transitions, but keeping the same number of tokens, it is possible to increase the period of the worst routing function arbitrarily. Therefore, bounding the period of the worst routing seems difficult, even for 2-bounded nets.

\section{References}

Baccelli, F., Cohen, G., Olsder, G., and Quadrat, J. (1992). Synchronization and Linearity. John Wiley \& Sons, New York. 
Bouillard, A., Gaujal, B., and Mairesse, J. (2005). Extremal throughputs in free-choice nets. In Ciardo, G. and Darondeau, P., editors, 26th International Conference On Application and Theory of Petri Nets and Other Models of Concurrency, LNCS. Springer-Verlag.

Carlier, J. and Chretienne, P. (1988). Timed Petri net schedules. In Advances in Petri Nets, number 340 in LNCS, pages 62-84. Springer Verlag.

Chretienne, P. (1983). Les Réseaux de Petri Temporisés. PhD thesis, Université Paris VI, Paris.

Cohen, G., Dubois, D., Quadrat, J., and Viot, M. (1985). A linear system-theoretic view of discrete-event processes and its use for performance evaluation in manufacturing. IEEE Trans. Automatic Control, 30:210-220.

Cohen, G., Gaubert, S., and Quadrat, J. (1998). Algebraic system analysis of timed Petri nets. In Gunawardena, J., editor, Idempotency. Cambridge University Press.

Desel, J. and Esparza, J. (1995). Free Choice Petri Nets, volume 40 of Cambridge Tracts in Theoretical Comp. Sc. Cambridge Univ. Press.

Gaubert, S. and Mairesse, J. (1999). Modeling and analysis of timed Petri nets using heaps of pieces. IEEE Trans. Aut. Cont., 44(4):683-698.

Gaujal, B. and Giua, A. (2004). Optimal stationary behavior for a class of timed continuous petri nets. Automatica, 40(9):1505-1516.

Gaujal, B., Haar, S., and Mairesse, J. (2003). Blocking a transition in a free choice net and what it tells about its throughput. J. Comput. Syst. Sci., 66(3):515-548.

Mairesse, J. and Vuillon, L. (1998). Optimal sequences in a heap model with two pieces. Liafa research report 98/09, Université Paris 7.

Recalde, L. and Silva, M. (2001). Petri net fluidification revisited: Semantics and steady state. European Journal of Automation APII-JESA, 35(4):435-449. 